\title{
Experimental Study of Developable Cones
}

\author{
Sahraoui Chaïeb and Francisco Melo \\ Departamento de Física de la Universidad de Santiago, Avenida Ecuador 3493, Casilla 307, Correo 2, Santiago, Chile \\ Jean-Christophe Géminard \\ Laboratoire de Physique de l'ENS de Lyon, 46 Allée d'Italie 69007 Lyon, France
}

(Received 21 August 1997)

\begin{abstract}
In this paper we investigate developable cones ( $d$-cones) topology. From profilometer measurements, we found that for a sample of a finite thickness the singularity is never pointlike but has a form of a crescent. Measurements of the sheet local curvature revealed that the $d$-cone tip is rejected by a distance which characterizes the singularity size. High deformations of the $d$-cone lead to a plastic regime equivalent to a decrease in the singularity size characterized from curvature and profile analysis. [S0031-9007(98)05464-7]
\end{abstract}

PACS numbers: 62.20.Fe, 03.40.Dz, 46.30.-i, 68.55.Jk

Strong deformations of membranes and thin shells span a wide range of scales. On the microscopic scale, quenched disorder in partially polymerized membranes and thermal fluctuations induce, without strain, a crumpling transition at the melting point, below which the membrane behaves like a 2D solid [1-3]. At the crumpling transition, partially polymerized vesicles look like dried prunes [1]. Some inorganic compounds such as nanotubes were observed in a phase that look similar to a crumpled sheet [4]. In much larger scales, in $(2+1)$-dimensional general relativity, defect-induced deformations of a two-dimensional sheet is characterized by a presence of conical singularities [5]. As Einstein's equations are similar to the Föpple-von Kármán (FvK) equations, they could leave some room for the occurrence of singularities, like the tip of a developable cone ( $d$ cone), which are surfaces with a shape of a cone but obtained from a plane isometrically, introduced in [6]. In intermediate scales, the stability of shells and thin elastic plates is of great importance in structure engineering and packaging material development [7]. Singularities that appear on a crumpled sheet, as a result of stress focusing, have been recently the subject of several investigations [7-10]. For instance, in the case of a crumpled sheet, $d$ cones were found to be the solution to FvK equations for large deflections [6]. A scaling analysis of the FvK equations showed that strain and deformation energy are located within the ridge region that separates conical singularities [9]. In practice, it was shown that singularity energy plays an important role in selecting characteristic lengths in a crumpled sheet: These lengths were shown to be the distance separating two $d$ cones [10]

In this Letter we present a systematic experimental study of an isolated $d$-cone topology and correspond it to a model experiment for situations where more than a singularity appears as in real crumpled paper. We investigate the variation of the local curvature along the $d$-cone generatrices in the concave and convex regions. Two regimes exist depending on the deformation experienced by the plate. We show that a $d$ cone obtained theoretically does not describe completely the crescent shape due to stretching and observed in all crushed sheets. However, for large deformations, the experimental $d$ cone approaches the theoretical one for zero thickness.

The $d$ cone is obtained on a thin circular plate by pushing a round tip $(0.5 \mathrm{~mm}$ diameter $)$ centered at the plate principal axis. In this study we used circular plates made from both 0.05- and 0.1-mm-thick sheets (copper, brass, steel, and transparencies); the results discussed are mainly from the $0.1-\mathrm{mm}$-thick sheets. In order to allow the $d$ cone to form, when pushing the tip, we keep the sheet border free to move in a circular rigid frame whose radius is $5 \%$ smaller than the sample radius $R_{f}$ (Fig. 1). The opening angle $\phi$ of the $d$ cone (defined as the angle between the horizontal and the cone generatrix) is varied by pushing the tip perpendicularly to the circular plate; the displacement $d$ is measured by a $10^{-2} \mathrm{~mm}$ precision micrometer. A miniature load cell is mounted under the pushing tip to allow force measurements. The pushing tip is mounted on a rigid 20-cm-long and 1-cm-thick steel pen-shaped cylinder. This bar is rigid enough to be inflexible when pushing the plate. A profilometric tip, mounted on the active part of a position sensor transducer, enables us to measure the sheet surface height with a precision of $10^{-2} \mathrm{~mm}$. Two motors allow the tip to scan the whole $d$-cone surface; first by moving the tip on a miniature automatic displacement guide mounted following the radial direction and second by rotating the frame around its axis. Both the radial and angular directions are marked in Fig. 1 as $(r)$ and $(\theta)$, respectively. The measurement precision of the $d$-cone opening angle is approximately $7 \times 10^{-4} \mathrm{rad}$. The whole system is run by a PC computer equipped with analogto-digital converter. In order to avoid stretching when a deformation $d$ is imposed, a part of the plate loses contact with the frame, giving rise to a concave region (Fig. 2), whose amplitude increases when increasing $d$ and whose location is randomly distributed on the plate. 


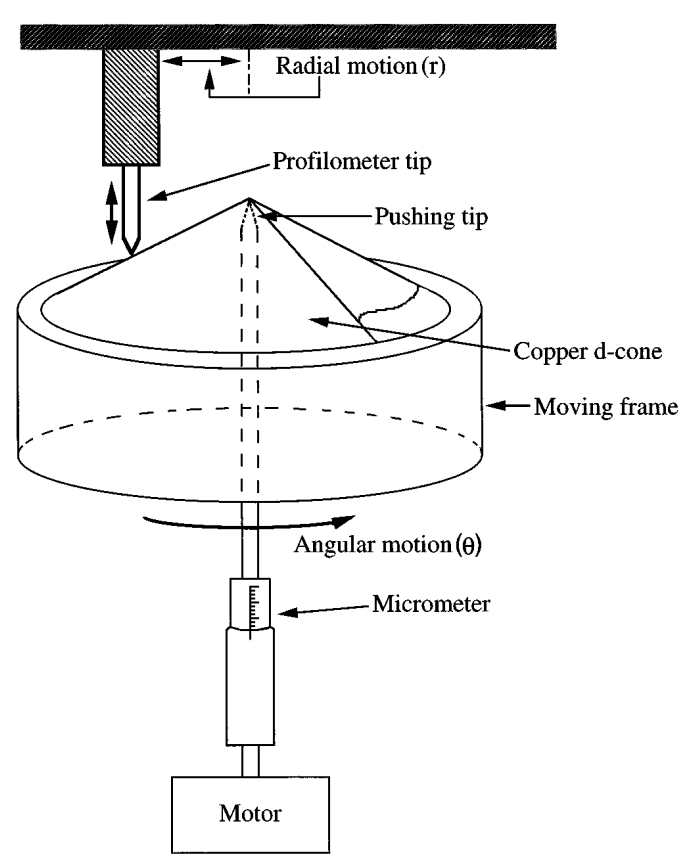

FIG. 1. The setup where the $d$ cone is performed and where the profilometric measurements are achieved.

If the pushing tip is deviated by a distance of the order of few millimeters from the center, the characteristics of the sheet deflection are not changed, but its nucleation occurs in the closest region from the pushing tip to the frame border. In some cases, two or four $d$ cones appear one in front of the other. Pushing the plate further, one of the deflection amplitudes increase, while the others disappear. Figure 2 is a top view of a $d$ cone obtained by this procedure. In the following, we will call the $d$-cone convex part the $(+)$ part and the concave part the $(-)$ part. The angle $2 \theta_{0}$ made by the $(-)$ part in the polar direction and over which the $d$ cone loses contact with the frame was found to be well-selected and independent on the tip displacement. The bright line shown in the figure defines a smaller angle and does not correspond to the angle in question. In Fig. 3, we present a set of profiles of a $d$ cone for two different $d$; each profile is obtained at varying distances $r$ from the singularity. The deflection

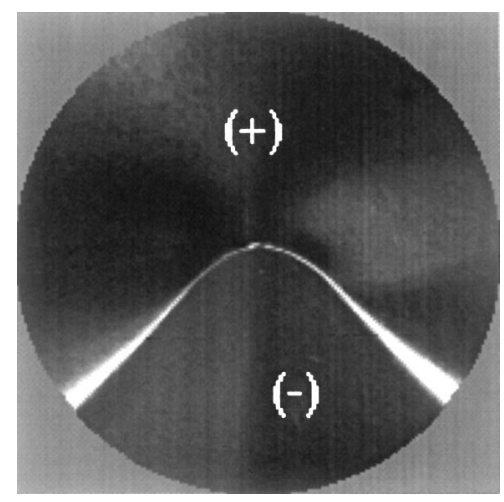

FIG. 2. A $d$ cone obtained on a copper sheet. Notice the bright line separating the concave and convex parts. amplitude is obtained by measuring the distance which separates the lowest point of the sheet $(-)$ part and the point where the plate loses contact with the frame. In Fig. 4, we plot the maximal vertical deflection angle $\phi_{\max }$ of the (-)part versus $\phi_{0}$ for a given distance $r$. The linear fit in Fig. 4 allows us to find the relation between $\phi_{\max }$ and $\phi_{0}$. In the following, we will show that the selection of both $\phi(\theta)$ and $\theta_{0}$ can be found by minimizing the $d$-cone bending energy. The general equation of a cone centered in $\mathrm{O}$, in cylindrical coordinates, is written as $z=r f(\theta)$. For convenience, we rewrite the parametric equation $z=r \tan \phi(\theta)$ and $r=R \cos \phi(\theta)$, where $R$ is the distance to the tip and $\theta$ is the polar angle. A cone corresponds to a given function $\phi(\theta)$, where $\phi$ is defined as above. For a given deformation $d^{*}=d / R_{f}=$ $\tan \phi_{0}$, where $d$ is the amount of the micrometer vertical displacement. If we write $\phi(\theta)=\phi_{0}$ for $|\theta|>\theta_{0}$ and

$$
\phi(\theta)=\phi_{0}+\epsilon\left(1+\cos \pi \frac{\theta}{\theta_{0}}\right) \text { for }|\theta|<\theta_{0},
$$

the function $\phi(\theta)$ defines then a cone that remains in contact with the circular frame for $|\theta|>\theta_{0}$. The $d$ cone is detached from the plate over an angle equal to $2 \theta_{0}$, which corresponds to the deflection. We assume that $d$ is small and that $\epsilon$ and $\phi_{0}$ are of the same order of magnitude. To the first order, the total curvature of the surface then reduces to $\kappa=\left(\phi+\phi^{\prime \prime}\right) / R$. The corresponding energy $E_{\kappa}$ (per unit of $R$ ) is

$$
\begin{gathered}
E_{\kappa}=\frac{K}{2} \int_{-\pi}^{\pi} \frac{\left(\phi+\phi^{\prime \prime}\right)^{2}}{R^{2}} R \sqrt{\cos ^{2} \phi+\phi^{\prime 2}} d \theta \\
\sim \frac{K}{2 R}\left[2 \pi \phi_{0}^{2}+4 \theta_{0} \phi_{0} \epsilon\right. \\
\left.\quad+\left(3 \theta_{0}-\frac{2 \pi^{2}}{\theta_{0}}+\frac{\pi^{4}}{\theta_{0}^{3}}\right) \epsilon^{2}\right] .
\end{gathered}
$$

For an unstretchable plate, the length $L$ of the corresponding line at $R=$ const must be equal to $2 \pi R$ so that

$$
\begin{aligned}
2 \pi R & =L=\int_{-\pi}^{\pi} R \sqrt{\cos ^{2} \phi+\phi^{\prime 2}} d \theta \\
& \sim\left[2 \pi-\pi \phi_{0}^{2}-2 \theta_{0} \phi_{0} \epsilon-\left(\frac{3 \theta_{0}}{2}-\frac{\pi^{2}}{2 \theta_{0}}\right) \epsilon^{2}\right] R .
\end{aligned}
$$

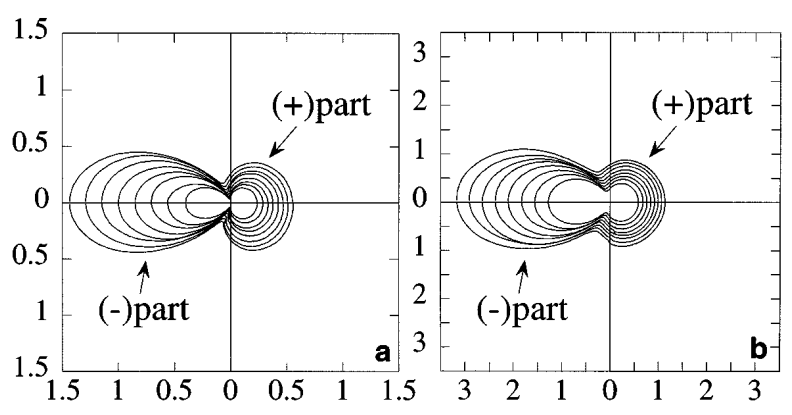

FIG. 3. The profile of the sheet in polar coordinates vs the angle $\theta$. (a) $d=1.41 \mathrm{~mm}$; (b) $d=5.48 \mathrm{~mm}$. The axes are in $\mathrm{mm}$. The different curves correspond to different distances to the $d$-cone tip. 


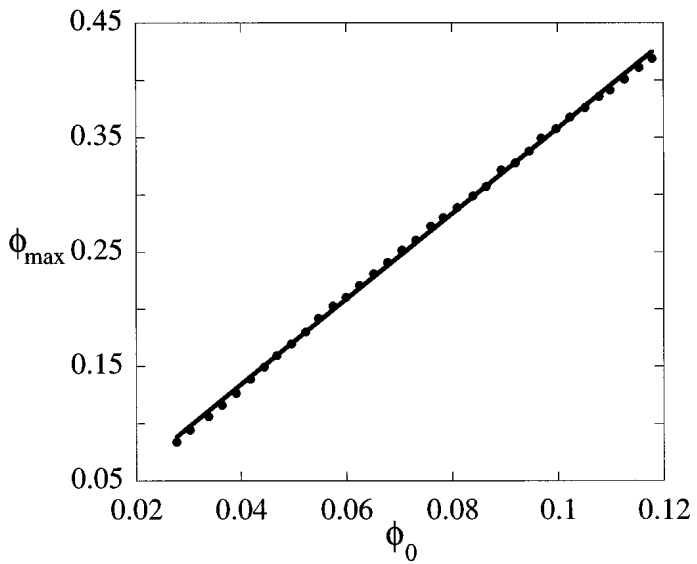

FIG. 4. The angle made by the $(-)$ part and the horizontal vs the angle made by the $(+)$ part and the horizontal $R_{f}=37.5$, and the distance to the singularity is $3 \mathrm{~mm}$.

Equation (2) gives $\epsilon$ as a function of $\phi_{0}$ and $\theta_{0}$. Replacing $\epsilon$ by its value in Eq. (2) and minimizing $E_{\kappa}$ with respect to $\theta_{0}$, one finds $2 \theta_{0} \sim 2.09 \mathrm{rad} \sim 120^{\circ}$ and $\epsilon \sim 1.38 \phi_{0}$. This last relation confirms our assumption that $\epsilon$ and $\phi_{0}$ are of the same order of magnitude. A solution to a similar problem gives $2 \theta_{0} \sim 140^{\circ}$ [11]. The theoretical value of the aperture angle $2 \theta_{0}$ is in good agreement with the experiment (Fig. 2) and is valid for small $d$. The aperture angle between the points where the plate loses contact with the frame is about $(110 \pm 5)^{\circ}$. The theoretical maximal deflection angle $\phi_{\max }=\phi(0)$ is proportional to $\phi_{0}$ and equals $\phi(0)=\left(\phi_{0}+2 \epsilon\right)=$ $3.76 \phi_{0}$. This result is in good agreement also with the experimental data, since the best fit in Fig. 4 gives $\phi(0)=3.73 \phi_{0}$. From Fig. 3, we notice that for large $r$ the profile is smooth and is very close to the one of a $d$ cone obtained by a bending energy minimization described previously. In contrast, within the singularity region the profile does not correspond to an ideal $d$ cone whose center is on the tip. The $d$-cone tip shift can be quantified by measuring what we called the $d$-cone anisotropy $\mathcal{A}$ defined as the ratio $[\zeta(\pi)-\zeta(\pi / 2)] /$ $\zeta(\pi)$, where $\zeta(\theta)$ is the height of the sheet measured at the polar angle $\theta$. From geometrical considerations $\mathcal{A}=\mathcal{A}\left(R_{f}, r, x_{s}\right)$, where $x_{s}$ is a distance by which the $d$-cone tip has shifted and which origin will be explained later. We measure experimentally $\mathcal{A}$ for different $r$ at a given $d$, we obtain $x_{s}$ [12]; $x_{s}$ decreases with an increase in $d$. On the other hand, we measured the (-)part local curvature for small $d$ vs $r$. In Fig. 5, we plot the (-)part local curvature versus $r$. The different curves correspond to different small $d$ ranging from 1.2 to $2.4 \mathrm{~mm}$. The curve is best fitted by a function of the form $1 /\left(r+r_{s}\right)$, and is not of the form $1 / r$ as expected for an ideal cone. In fact, $r_{s}$ can be interpreted as the shift of the coordinates origin of the cone (-)part. In Fig. 6, we plot both $r_{s}$ and $x_{s}$ versus $d$. We notice that as we increase $d$, both quantities decrease quickly. In fact, $x_{s}$ and $r_{s}$ are indirect measurements of the singularity size. For small

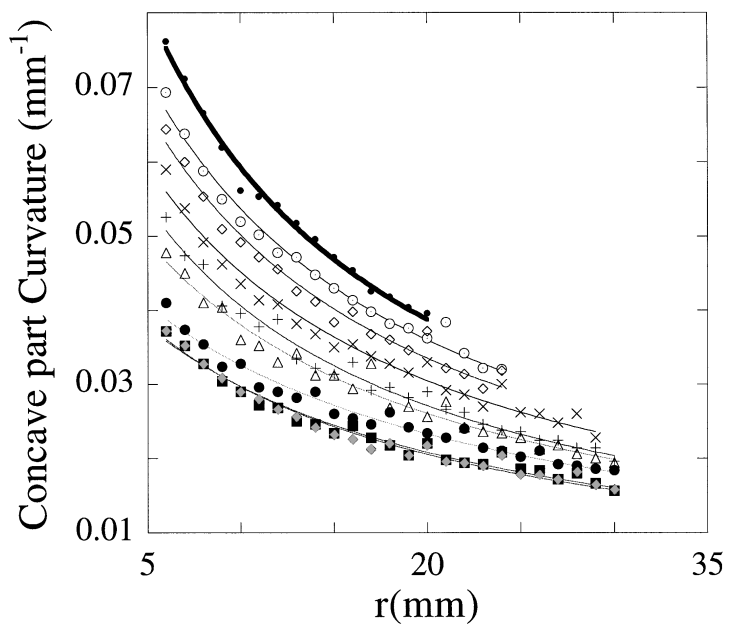

FIG. 5. The (-) part local curvature vs the distance to the singularity, and for different small $d$. The lines are best fit to the function of the form $1 /\left(r+r_{s}\right)$. The curves range from small to large deformation.

$d$, plate curvature is distributed over the whole sample and the singularity size is equal to the sample radius. However, as we increase $d$, the deformation is focused and the singularity size decreases. The singularity size region can also be observed in Fig. 2; the sample avoids formation of pointlike singularity by making a linelike singularity which corresponds to the thinner part of the line separating the $(+)$ part and the (-)part of the cone in Fig. 2. This line that is hyperbolic shaped can be obtained by intersecting a plane with a cone at the tip micrometer and parallel to a generatrix. This cone is centered at a distance $x_{s}$ from the micrometer tip along its generatrix along the (+)part. The thin line obtained by the plane cut corresponds then to the crescent shape observed in crumpled papers. We have measured the curvature of the crescent shown in Fig. 2 and found that it decreases with $d$ [12]. In Figs. 7(a) and 7(b), we plot the (-)part and the ridge local curvature vs $r$, respectively, and for

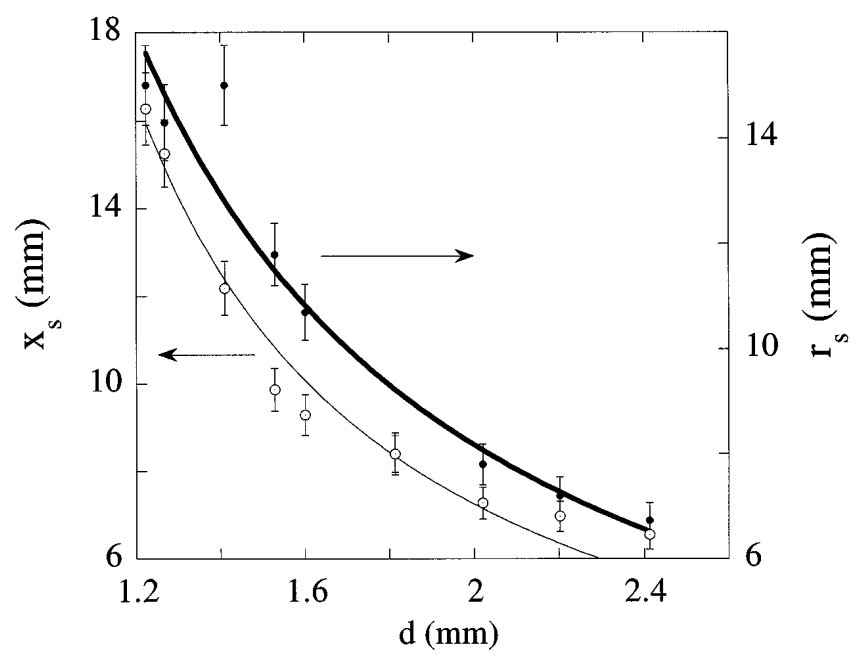

FIG. 6. The shift distance $r_{s}$ and the singularity displacement $x_{s}$ as a function of $d$. (•) correspond to $r_{s},(\circ)$ correspond to $x_{s}$. 
large $d$. The fit to the curvature is no longer of the form $1 / r$. Instead, it varies like $C_{0} e^{\left(-r / d_{c}\right)}$, where $d_{c}$ is a cutoff distance. In the case of the (-)part 7(a), $d_{c}$ does not vary with $d$. Whereas on the ridge 7(b), $d_{c}$ decreases with $d$. In Fig. 7(a), the cutoff distance is constant because the crescent due to the permanent scar appears on the ridges only. The plastic deformation is felt on the border between the (-)part and the (+)part of the $d$ cone where the folding occurs and the stress is maximum. In fact, by looking at the ridge curvature, one can notice that $d_{c}$ reaches a value of about $5 \mathrm{~mm}$ at which a permanent scar appears. This value corresponds also to the curvature at which the yield limit of a 0.1-mm-thick copper sheet is exceeded [10].

The deviation of the curvature from $1 / r$ behavior to an exponential is due to the fact that at large $d$ the yield limit of the material is exceeded and stretching starts to be important with respect to pure bending. As a first approximation, we assume that the (-)part is an isolated stripe. By further pushing the plate beyond the yield limit, the stripe starts to bend and the region near the singularity suffers stretching. Following [13], if we include stretching in the energy balance, we find that the stripe local curvature decreases like an exponential, and the cutoff distance decreases by increasing the height of the sheet, that is, by pushing the plate [9]. The curve giving the curvature versus the distance for a $d$ cone made of a $0.05-\mathrm{mm}$-thick sheet gives a cutoff distance that is half of the one for a 0.1 -mm-thick sheet. We noticed no qualitative changes between the two plates, and we believe that the cutoff distance is a linear function versus $h$.

Studying local properties of a $d$ cone made of a thin circular plate has revealed the origin of the crescent shape observed on crumpled sheets. For small plate deformations, the local curvature along the generatrices follows the known power law $1 / r$ as for a cone of revolution. By an increase in the deformation, the local curvature along the generatrices is no longer of the form $1 / r$ but follows an exponential law due to stretching near the tip. By measuring the anisotropy of the deformed plate profile, we found that the $d$-cone tip moved by a distance $x_{s}$, which depends on the deformation $d$ and characterizes the singularity size. For small $d$, the

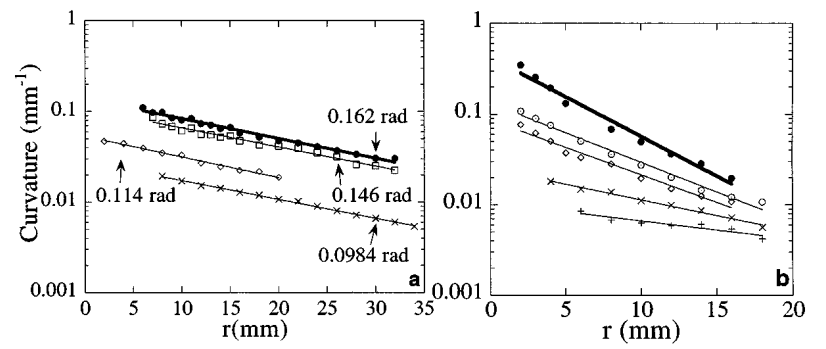

FIG. 7. The $d$-cone local curvature vs the distance $r$ from the singularity for different $d$. (a) The (-) part local curvature, $d_{c}$ is constant and equal to $20 \mathrm{~mm}$. (b) The ridge local curvature, $d_{c}$ decreases when increasing $d$. In the figure we report the different value of $\phi$ instead of $d$. singularity region is spread over the sample, whereas, for large $d$, the curvature is focused and the singularity size is reduced to a small region, characterized by the crescent shape [12]. The decrease in the singularity size with deformation appears as analogous to the focusing of topological defects under constraints [14]. The singularity hyperbolic shape is found by intersecting a plane with a theoretical cone. This cone is centered at the distance $x_{s}$ from the pushing tip. The plastic transition is the appearance of a crescent shape on the sheet and the focusing of the ridge curvature [15]. Although crumpled vesicles have been observed [1], no systematic local study of the surface of a crumpled vesicle has been performed [16]. Profilometry using laser beam or magnetic beads on the surface of a crumpled vesicle can complement freeze fracture microscopy experiments usually used to probe vesicles in suspensions.

We thank E. Cerda for discussions and F. Vivanco for technical assistance. This work was financed in part by the DICyT of the University of Santiago Grant No. 049631CH, and by a Catedra Presidencial en Ciencias.

[1] M. Mutz, D. Bensimon, and M. J. Brienne, Phys. Rev. Lett. 67, 923 (1991).

[2] Y. Kantor, M. Kardar, and D. R. Nelson, Phys. Rev. Lett. 57, 791 (1986); Statistical Mechanics of Membranes and Surfaces, edited by D. Nelson, T. Piran, and S. Weinberg (World Scientific, Singapore, 1989).

[3] D. R. Nelson and L. Peliti, J. Phys. (Paris) 48, 1085 (1987); M. Paczuski, M. Kardar, and D. R. Nelson, Phys. Rev. Lett. 60, 2638 (1988); D. Morse, T. Lubensky, and G. Grest, Phys. Rev. A 45, R2151 (1992); D. Bensimon, D. Mukamel, and L. Peliti, Europhys. Lett. 18, 269 (1992); R. Attal, S. Chaïeb, and D. Bensimon, Phys. Rev. E 48, 2232 (1993).

[4] M.S. Spector, E. Naranjo, and J. A. Zasadzinski, Phys. Rev. Lett. 73, 2867 (1994); R. R. Chianelli, E. B. Prestige, T. A. Pecoraro, and J.P. DeNeufville, Science 203, 1105 (1979).

[5] S. Deser, R. Jackiw, and G. 't Hooft, Ann. Phys. 152, 220 (1984).

[6] Y. Pomeau, C. R. Acad. Sci. Ser. I 320, 975 (1995); M. Ben Amar and Y. Pomeau, Proc. R. Soc. London A 453, 729 (1996).

[7] A. Lobkovsky, S. Gentges, Hao. Li, D. Morse, and T. A. Witten, Science 270, 1482 (1995).

[8] T. A. Witten and H Li, Europhys. Lett. 23, 51 (1993); E. M. Kramer and T. A. Witten, Phys. Rev. Lett. 78, 1303 (1997).

[9] A. Lobkovsky, Phys. Rev. E 53, 3750 (1996).

[10] S. Chaïeb and F. Melo, Phys. Rev. E 56, 4736 (1997).

[11] E. Cerda and L. Mahadevan (private communication).

[12] S. Chaïeb and F. Melo (unpublished).

[13] L. Landau and E. Lifchitz, Théorie de l'élasticité (Mir, Moscow, 1967), p. 87.

[14] P. Oswald (private communication).

[15] A plastic deformation has been checked by force measurements.

[16] D. Bensimon (private communication). 